\title{
41st ANNUAL SASKATCHEWAN CHRISTMAS BIRD COUNT - 1982
}

Compiled by MARY I. HOUSTON, 863 University Drive, Saskatoon, Saskatchewan. S7N 0J8

The weather over the 1982 Christmas Bird Count period (18 December 1982 to 2 January 1983) cooperated with bird counters, enabling counts to be taken in 63 localities. The total number of species recorded on count days was 77 , with 2 additional species seen during count period but not on count days. This is the 4th highest count over the 41 years $(79+4$ in $1974 ; 78+4$ in 1981 ; $76+5$ in 1979). Saskatoon had the highest number of species over count period with $35(34+1$ additional). Fort Walsh reported $32(31+1)$; Regina, 30 $(28+2)$; Squaw Rapids, 28 and Gardiner Dam, 27.

Three new species were recorded: 1 Common Loon was seen at Gardiner Dam and 1 Killdeer and 1 McCown's Longspur were seen at Govenlock. This brings the all time total of Count Day species to 136 plus 2 races and 6 additionals seen during Count Period but not on Count Day.

Only Fort Walsh reported Hoary Redpolls (15). Common Redpolls were low being reported from only 9 of the 63 localities. Bohemian Waxwings were down both in number of localities reporting and in numbers seen; on the other hand numbers of Cedar Waxwings increased with 9 reports. Saskatoon and Moose Jaw had 75 each and Regina, 36. Cedars outnumbered Bohemians in Regina and Skull Creek, and only Cedars were seen in Moose Jaw. Pine Grosbeak numbers were down, with only 12 localities reporting them.

Exceptionally large flocks of Snow Buntings (reported from 54 of the 63 localities) were seen at Govenlock (6183), Kutawagan Lake (9483), Last
Mountain Lake (12944) and Raymore (33935).

After the list of participants from the 63 localities, Table 1 gives the Coverage and Weather; Table 2 gives species reported in more than 3 locations and gives numbers of each species seen in each locality on count day, with numbers for additional species seen during count period indicated with a + sign; Table 3 gives numbers and locations of species seen in 3 or less locations during Count Period; Table 4 gives locations and numbers of birds not positively identified as to species.

\section{Count Areas and Participants}

Names of compilers in italics.

1. ASSINIBOIA. Carol and Ed Bearss, Murray Brunt, John G. Burgeson, Alex Duxbury, Gordon Forsythe, Cecil T. Hayward, Nelson Lamb, Don Lemond, Don Lewis, Ted McMorrine, Wilf Prentice, Ken Schuweiler.

2. BIGGAR. Harvey Renaud, Wayne Renaud, Guy Wapple, Robert Wapple.

3. BIG GUL_LY CREEK. Wayne Harris, Sheila Lamont, Tom Lamont.

4. BIRCH HILLS. Terry Toews, Don Weidle.

5. BRIGHTWATER RESERVOIR. Linda Lahey, Alan R. Smith.

6. BROADVIEW. Doug Francis, Joe Grimeau, Pat Kern.

7. BROOKSBY. Bert and Vi Ross

8. CROOKED LAKE. DOn Weidl, Tony Weidl. 
1. ASSINIBOIA

2. $B I G G A R$

3. BIG GULLY CREEK

4. BIRCH HILLS

5. BRIGHTWATER RESERVOIR

6. BROADVIEW

7. BROOKSBY

8. CROOKED LAKE

9. DALMENY

10. DILKE

11. ELBOW

12. ENDEAVOUR

13. FORT QU'APPELLE

14. FORT WALSH

15. GARDINER DAM

16. GLAMIS-WISETON

17. GOODSOIL

18. GOOD SPIRIT LAKE

19. GOVENLOCK

20. GRAND CENTRE (Sask.-Alta.)

21. HARRIS

22. HUMBOLDT

23. INDIAN HEAD

24. KELVINGTON

25. KELVINGTON

26. KENASTON

27. KIILLSQUAW LAKE

28. KINDERSLEY

29. KUTAWAGAN LAKE

30. LA RONGE

31. LAST MOUNTAIN LAKE

32. LEADER
33. LEASK

34. LOON LAKE

35. LOVE-TORCH RIVER

36. LUSELAND

37. MAIDSTONE BRIDGE

38. MELFORT

39. MOOSE JAW

40. NAICAM

41. NIPAWIN

42. NISBET FOREST

43. PRINCE ALBERT

44. PRINCE ALBERT

45. RAYMORE

46. REGINA

47. ROSE VALLEY

48. ST. WALBURG

49. SASKATCHEWAN FORKS

50. SASKATOON

51. SCOTT

52. SEMANS

53. SKULL CREEK

54. SOMME

55. SPRING VALLEY

56. SQUAW RAPIDS

57. TISDALE

58. TOGO

59. WASKESIU

60. WEBB

61. WHITE BEAR

62. WHITEBEECH

63. WOLSELEY SOUTH

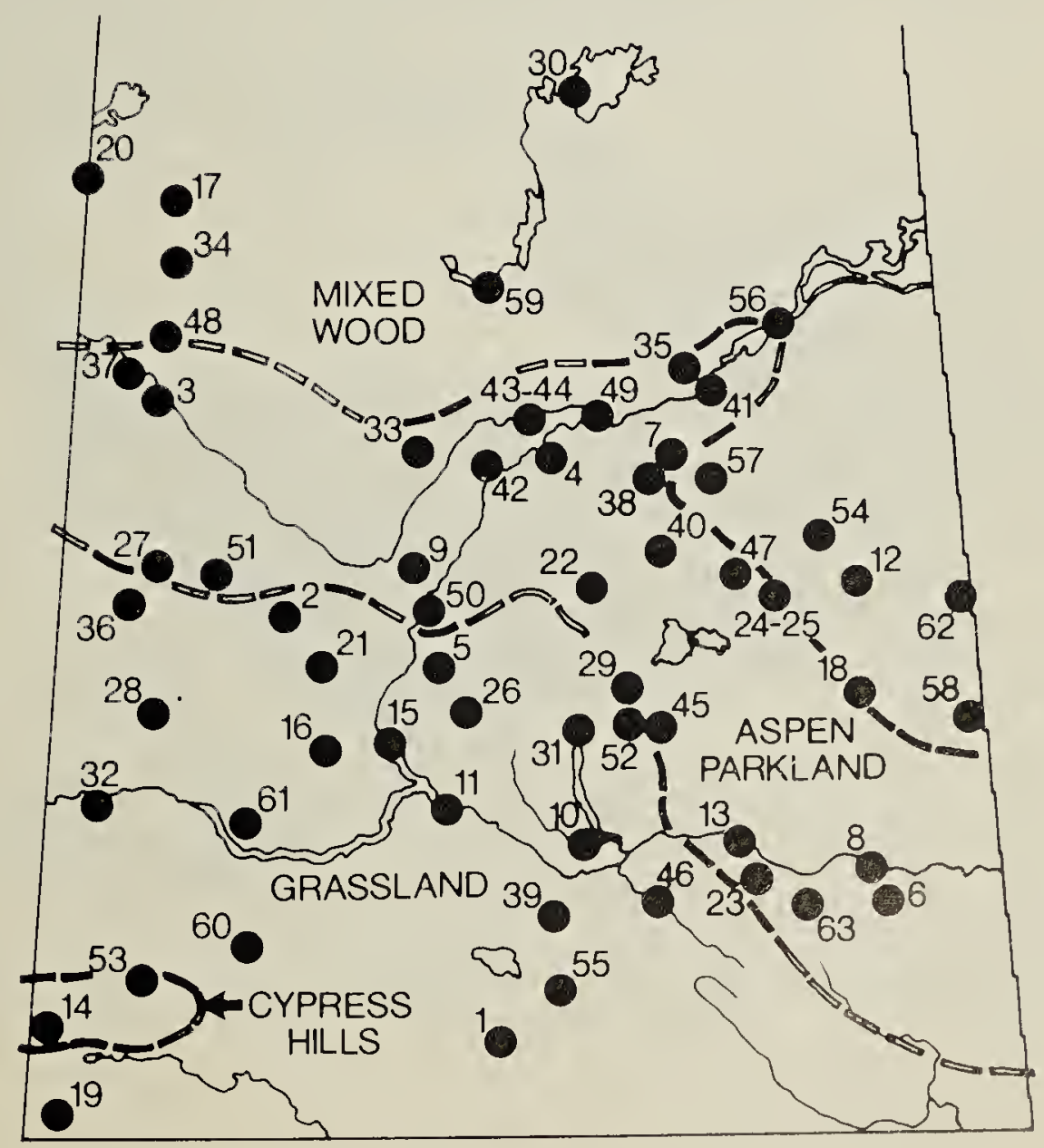


9. DALMENY. Lorne Sperling, Lloyd Sperling, Mara Sperling.

10. DILKE. Margaret Belcher, Doug Laing.

11. ELBOW. Ron Jensen, Dan Mclntosh, Alan R. Smith.

12. ENDEAVOUR. Norman Harris.

13. FORT QU'APPELLE. Doug Evans, Alma Hiebert, Selma Hiebert, David Hooper, Ron Hooper, Alice Laing, Lois Lamontagne, Victor Lamontagne, Don McDougall, Fern McDougall, Raymond Mlazzar, Lorne Rowell, Kay Ruard, Eileen Smith, Clinton Wilde, Flo Wilde.

14. FORT WALSH. Chris Adam, Dixie Black, Bob Godwin, Wayne Harris, Dale and Paule Hjertaas, Bob Kreba, Sheila Lamont, Kathy Meeres, Wilkes Parsonage, Wayne Renaud, John Triffo, Guy Wapple, Robert Wapple, Jack Wilkinson.

15. GARDINER DAM. Wayne Harris, Sheila Lamont, Wayne Renaud, Guy Wapple, Robert Wapple.
16. GLAMIS-WISETON. Brian $H$ Jones, Grev. L. Jones.

17. GOODSOIL. Catherine, David and Richard Morton.

18. GOOD SPIRIT LAKE. Bill Anaka, Joyce Anaka.

19. GOVENLOCK. Chris Adam, Tom Buchanan, Wayne Harris, Dale Hjertaas, Paule Hjertaas, Bob Kreba, Sheila Lamont, Wayne Renaud, Bill Russon, John Triffo, Guy Wapple, Robert Wapple.

20. GRAND CENTRE (Sask.-Alta.) J. F. Roy

21. HARRIS. Guy Wapple, Robert Wapple.

22. HUMBOLDT. Ed Brockmeyer, Dwayn Saretsky.

23. INDIAN HEAD. Susan Adams, Les Anderson, Betty and Cec Ashmore, Marg Barclay, Isabel and Peter Barrett, Margo and Vic Beaulieu, Yvonne Brown, Mavis and Roger Gray, Jim Halford, Don Hayward,

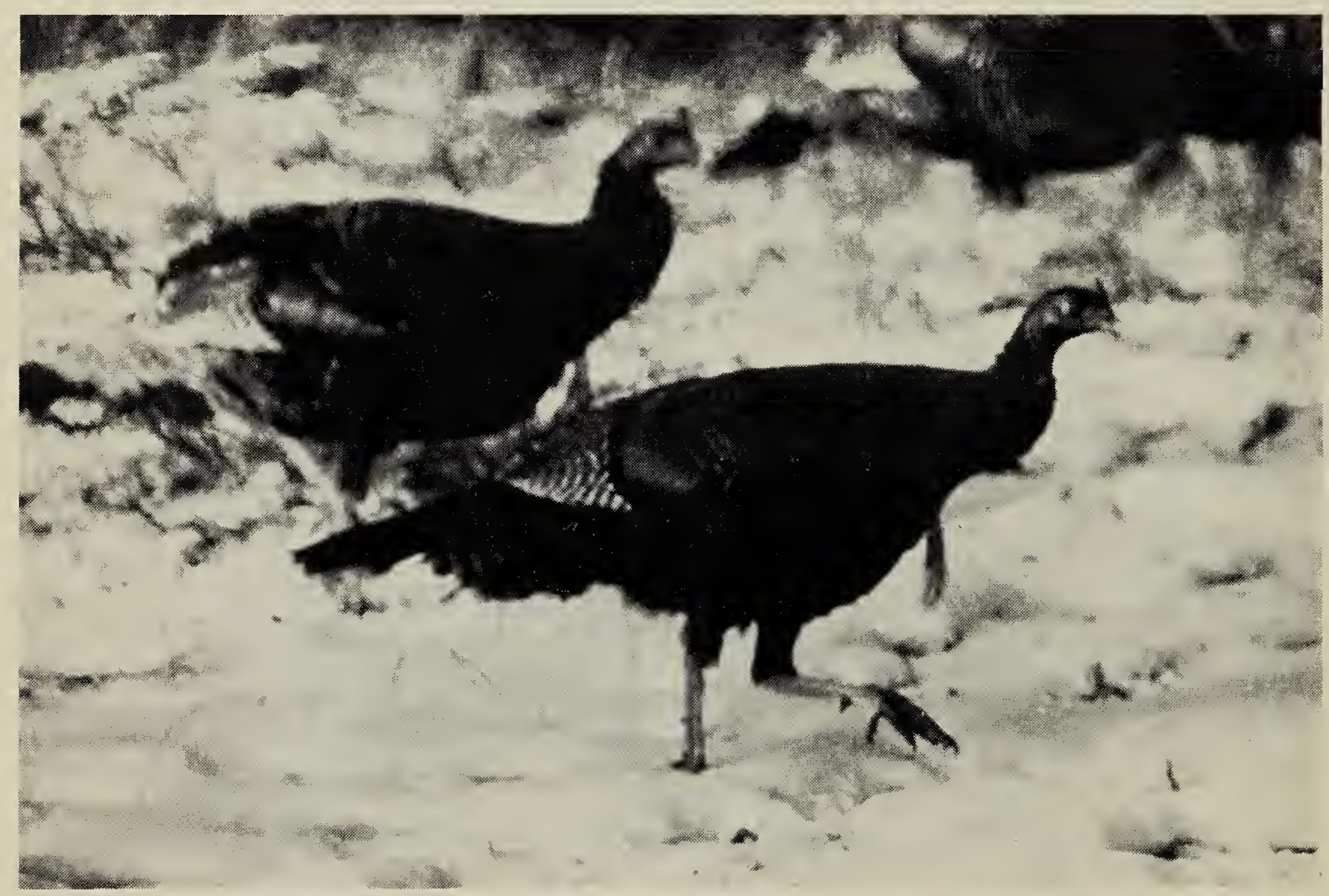


Gordon Howe, Roy McLaughlin, Doris Pals, Mariel Peterson, Lloyd Peterson, Marg Quigley, Joan and Lorne Scott, Gertrude Scott, Fred Skinner, Mary Skinner, Ron Thompson family, Ann and Gordon Willerth, Shaun Williamson.

24. KELVINGTON, Dianne Sloan.

25. KELVINGTON. James Donovan.

26. KENASTON. P. Lawrence Beckie.

27. KILLSQUAW LAKE. Linda Lahey, Alan R. Smith.

28. KINDERSLEY. Jean Harris.

29. KUTAWAGAN LAKE. Len Harding, Wayne Harris, Sheila Lamont.

30. LA RONGE. Jim Paul.

31. LAST MOUNTAIN LAKE. Wayne Harris, Sheila Lamont.

32. LEADER. Daisy D. Meyers.

33. LEASK. Muriel Carlson, Page Porter.

34. LOON LAKE. P. Davis, G. Pedersen, F. Scott, M. Williams.

35. LOVE-TORCH RIVER. Bert Dalziel, Joan Dalziel, Bruce Donovan.

36. LUSELAND. E. J. Finley, K. B. Finley, K. J. Finley, B. Holton.

37. MAIDSTONE BRIDGE. Wayne Harris, Sheila Lamont, Tom Lamont.

38. MELFORT. Genevieve Belliveau, Harry Belliveau, Bill Bristol, Philip Curry, Glen Galloway, Freida Markland, Tom Smith, Kathy Stevenson.

39. MOOSE JAW. Alice and Carl Ellis, Doug Francis, Alta and Don Fyvie, Al Guernsey, Edith Kern, Pat Kern, Eve King, Leith and Cy Knight, Alice and Gordon Silversides, Arie and Henry Van Dorland, Gus and Mary Zado.

40. NAICAM. Julie Jensen, Ron Jensen.
41. NIPAWIN. Bert Dalziel, Joan Dalziel, Bruce Donovan.

42. NISBET FOREST. Muriel Carlson, Gail Paine, Vetha Paine.

43. PRINCE ALBERT, John Burt, Pamela Burt.

44. PRINCE ALBERT. Kim Hruska, Dr. and Mrs. H. A. Martinson.

45. RAYMORE. Greta Harris, Wayne Harris, Sheila Lamont.

46. REGINA. Chris Adam, Jessie Bailey, Margaret Belcher, Tom Beveridge, Frank Brazier, Kirsten Costain, Joanne Edwards, M. Edwards, L. Eley, Tony Lang, Bob Luterbach, Ron Mendelsohn, Gillian Minifie, Irene Pierson, Bill Puscus, Brian Rainey, Tom Riffel, Bill Russon, Diane Secoy, Allan Smith, Frank Switzer, Ian Switzer, R. Tegart, R. L. Tegart, John Triffo. Chris Adam and Bob Kreba.

47. ROSE VALLEY. Julie Jensen, Ron Jensen.

48. ST. WALBURG. Nigel Caulkett.

49. SASKATCHEWAN FORKS. Terry Toews, Don Weidl.

50. SASKATOON. Mark Abley, Juhachi Asai, Carol Balog, Annie Beer, Raymond Bisha, Muriel Carlson, Debra Cohen, Eric Densem, Murray Doell, Chris Escott, Barry Evenson, Hartley Fredeen, Muriel Galloway, Mary Gilliland, Bob Godwin, Bernie Gollop, Bev Haug, Del Haug, David Houston, Mary Houston, Stuart Houston, Louise Klaassen, Catherine Laratte, Ken Lumbis, Kathy Meeres, Stew Meeres, Betty Mundy, Jim Mundy, Laurie Murison, Tom Murison, Bert McBride, Cameron McBride, Richard McBride, Jean McQueen, Don McRobbie, Jo McRobbie, Mickey Narun, Bill Nickel, Lynn Oliphant, Jim O'Neil, Pat O'Neil, John Polson, Adam Schmidt, 
Charlie Simpson, Jim Slimmon, Alan Smith, Gerald Smith, Mary Strickland, Shannon Voysey, Jim Wedgwood, Elda Windsor, Henry Windsor.

51. SCOTT. Guy Wapple, Robert Wapple.

52. SEMANS. Tony Lang, Sarogini Ramnarine, Lloyd S. Saul, Jane Yee.

53. SKULL CREEK. Eileen Bennetto, James Bennetto, Marj Mann, Jr., Murina Schuler.

54. SOMME. Margaret Back, Stan Back, Alice Billeter, Edwin Billeter, Shirley Billeter, David Black, Florence Chase, Ian Chase, Jim Chase, Donald Hooper, Eldon Hooper, David Osecki.

55. SPRING VALLEY. Allan Bogdan, Flossie Bogdan, Nick Bogdan.

56. SQUAW RAPIDS. Wayne Harris, Sheila Lamont, Wayne Renaud, Guy Wapple, Robert Wapple.
57. TISDALE. Joyce Mohr.

58. TOGO. Bert and Doris Franklin, Phil and Jean Hern, Walter Krupp, Cusie and Wanda May, Arch and Elizabeth Parker, Mary and Dick Smith, Howard and Donelda Wilson, Orville and Elaine Wilson.

59. WASKESIU. Suzanne Henry, Anne Landry, Bradley and June Muir, John Pollock, Kathy Ryhorchuk, Glen and Lorraine Schmidt, Kathy Stefanuk, Pat Strutt, Jackie and Mervyn Syroteuk.

60. WEBB. Catherine Dumouchel, Jim Gibson, Stan Greenwood, Lois Nisbet, Jack Ricou.

61. WHITE BEAR. Sig Jordheim, Carl Maalerud.

62. WHITEBEECH. Tracy Hutchinson, Ida Wotherspoon, Lindsay Wotherspoon.

63. WOLSELEY SOUTH. Dale Chay, Don Hayward.

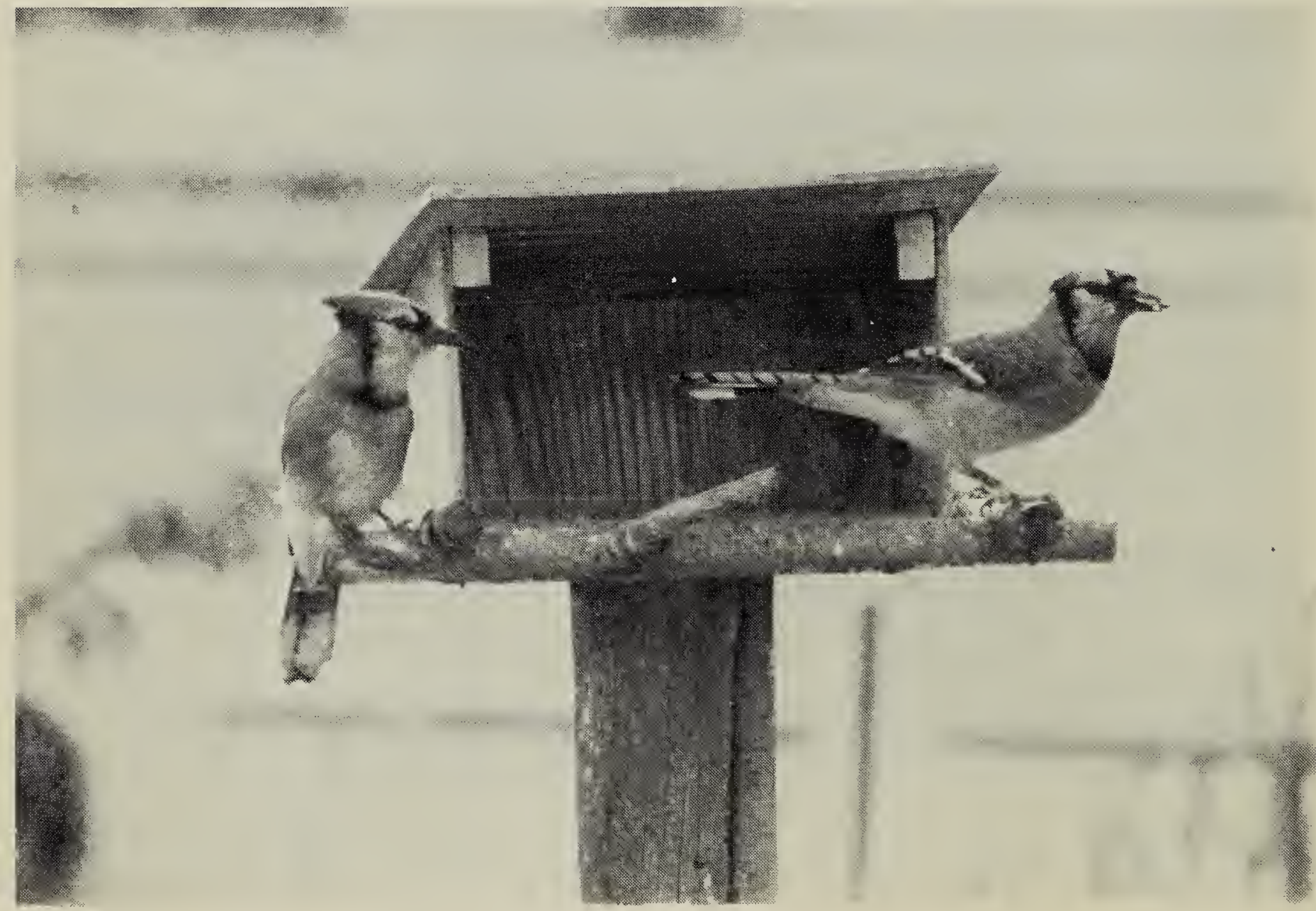


Table 1. CHRISTMAS COUNT COVERACE AND WEATHER CONDITIONS.

\begin{tabular}{|c|c|c|c|c|c|c|c|c|c|}
\hline 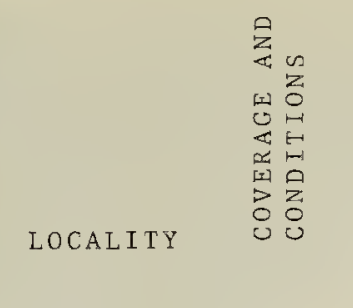 & 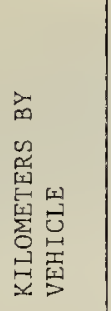 & 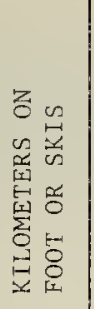 & 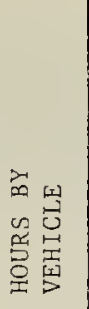 & 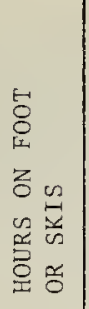 & 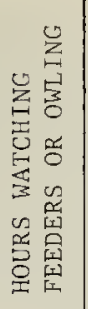 &  & 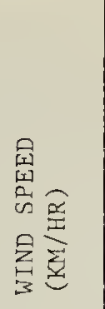 & 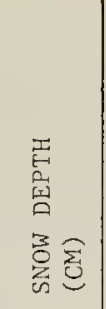 & $\frac{d}{c}$ \\
\hline \multirow{5}{*}{$\begin{array}{l}\text { ASSINIBOIA } \\
\text { BIGCAR } \\
\text { BIG CULLY CREEK } \\
\text { BIRCH HILLS } \\
\text { BRICHTWATER RESERVOIR }\end{array}$} & 33.5 & 1 & 2 & & & $-6 /-4$ & $10-15$ & $7-10$ & mostly cloudy, light snow \\
\hline & 212 & 26 & 8 & 10.5 & & $-13 /-7$ & $20-30$ & $10-20$ & partly cloudy, light snow \\
\hline & 145 & 10 & 6 & 4 & 1.5 & $-8 /-3$ & $0-5$ & $8-23$ & overcast, partly cloudy \\
\hline & 194 & 3 & 5.5 & 1 & 1.5 & $-6 /-8$ & $10-15$ & $10-15$ & overcast, light snow \\
\hline & 55 & .5 & 3 & .5 & & $-10 /-12$ & $0-5$ & $10-20$ & overcast \\
\hline \multirow{5}{*}{$\begin{array}{l}\text { BROADVIEW } \\
\text { BROOKSBY } \\
\text { CROOKED LAKE } \\
\text { DALMENY } \\
\text { DILKE }\end{array}$} & 210 & 1 & 5.5 & & 3 & $-4 /-10$ & light & 18 & cloudy, clearing, light snow \\
\hline & 10 & 5 & 1 & 3 & & $-3 /-4$ & $0-5$ & $0-5$ & overcast \\
\hline & 80 & 3 & 4 & 1 & & $-19 /-16$ & 5 & $15-20$ & mostly cloudy \\
\hline & & 16 & & 5 & & -16 & $22-26$ & $2-50$ & overcast, light snow \\
\hline & 137 & 7 & 6 & 2.5 & & $-5 /-8$ & $15-5$ & $2-30$ & cloudy, clearing, cloudy \\
\hline \multirow{5}{*}{$\begin{array}{l}\text { ELBOW } \\
\text { ENDEAVOUR } \\
\text { FORT QU'APPELLE } \\
\text { FORT WALSH } \\
\text { GARD INER DAM }\end{array}$} & 80 & 5 & 4 & 4 & & $-10 /-5$ & $0-10$ & $0-3$ & overcast \\
\hline & 9 & 2 & 2 & 3 & & $-20 /-2$ & calm & $20-30$ & cloudy, clearing \\
\hline & 240 & & 14 & & 1 & $-5 /-2$ & $15-30$ & $5-10$ & overcast \\
\hline & 225 & 100 & 21.5 & 41.5 & 2 & $-4 /+1$ & $20-30$ & $0-10$ & clear, partly cloudy \\
\hline & 267 & 23 & 16 & 10 & & $-8 /-1$ & $0-20$ & $0-5$ & clear, clouding over \\
\hline \multirow{5}{*}{$\begin{array}{l}\text { CLAMIS-WISETON } \\
\text { COODSOIL } \\
\text { GOOD SPIRIT LAKE } \\
\text { GOVENLOCK } \\
\text { CRAND CENTRE }\end{array}$} & 30 & 5 & 1 & 2 & & $-5 /-3$ & $0-5$ & $6-15$ & mostly clear \\
\hline & 30 & .5 & & & 6 & $-5 /-15$ & $0-5$ & $10-25$ & $?$ \\
\hline & 92 & 13 & 5 & 3.5 & 1 & -7 & $\mathrm{calm}$ & $12-15$ & overcast \\
\hline & 173 & 42 & 15 & 16 & & $-3 /+3$ & $2-10$ & $0-2$ & mostly clear \\
\hline & 85 & 1 & 2.5 & .5 & & -16 & $24-48$ & 30 & overcast, light to heavy snow \\
\hline \multirow{5}{*}{$\begin{array}{l}\text { HARRIS } \\
\text { HUMBOLDT } \\
\text { INDIAN HEAD } \\
\text { KELVINCTON } \\
\text { KELVINCTON }\end{array}$} & 202 & 10 & 5 & 4 & & $-6 / 0$ & $15-20$ & $10-30$ & most 1 y cloudy \\
\hline & 35 & 15 & .5 & 2.5 & & $-9 /-6$ & 0 & 20 & overcast, clearing \\
\hline & 75 & 13 & 5.5 & 10 & & -7 & 10 & 20 & overcast \\
\hline & 25 & & .5 & & 5 & $-10 /-7$ & 0 & 20 & overcast, light fog \\
\hline & $\overline{10}$ & 2 & 1 & .5 & 1.5 & $-10 /-12$ & calm & $20-23$ & overcast \\
\hline \multirow{5}{*}{$\begin{array}{l}\text { KENASTON } \\
\text { KILLSQUAW LAKE } \\
\text { KINDERSLEY } \\
\text { KUTAWAGAN LAKE } \\
\text { LA RONGE }\end{array}$} & 25 & & 7 & & & -8 & $\mathrm{calm}$ & $?$ & clear \\
\hline & 98 & 1 & 5.5 & .5 & & -15 & 0 & $10-20$ & overcast \\
\hline & 46 & 1.5 & & & & $-8 /-3$ & light & $7-15$ & partly cloudy \\
\hline & 210 & 6 & 7 & 2 & & $-16 /-10$ & $3-1 \overline{6}$ & $2-20$ & heavy snow, overcast \\
\hline & 40 & 11 & 2 & 3 & & $-11 /-8$ & $10-18$ & $30-50$ & overcast \\
\hline \multirow{5}{*}{$\begin{array}{l}\text { LAST MOUNTAIN LAKE } \\
\text { LEADER } \\
\text { LEASK } \\
\text { LOON LAKE } \\
\text { LOVE-TORCH RIVER }\end{array}$} & 180 & 5 & 7 & 2 & & $-15 /-9$ & $2-8$ & $2-20$ & light fog, clearing \\
\hline & 1 & 5 & .5 & 2 & 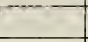 & $-10 /+2$ & $0-\overline{30}$ & $2-7$ & clear \\
\hline & & 20 & & 5 & & $-11 /-7$ & 0 & $20-25$ & overcast \\
\hline & 20 & 2 & .5 & 1 & 8 & $-15 /-10$ & $15-20$ & $10-15$ & overcast, moderate snow \\
\hline & 70 & 2 & 2 & 1 & 2 & $-5 /-10$ & $0-5$ & $10-20$ & overcast \\
\hline \multirow{5}{*}{$\begin{array}{l}\text { LUSELAND } \\
\text { MAIDSTONE BRIDCE } \\
\text { MELFORT } \\
\text { MOOSE JAW } \\
\text { NAICAM }\end{array}$} & 142 & 18 & 3 & 5 & 2 & $-8 /-2$ & $0-35$ & $20-30$ & clear, clouding aver \\
\hline & $\$ 50$ & 10 & 6 & 4 & 2. & $-15 /-5$ & $0-8$ & $5-20$ & overcast, light snow \\
\hline & 25 & 5 & 1 & 3 & & $-13 /-8$ & $?$ & $3-8$ & overcast \\
\hline & 107 & 10 & 5 & 9 & 1 & $-3 / \quad 0$ & $5-13$ & 7 & mostly clear \\
\hline & 80 & 7 & 4 & 4 & & $- 1 2 \longdiv { - 1 5 }$ & $?$ & $?$ & partly cloudy \\
\hline \multirow{5}{*}{$\begin{array}{l}\text { NIPAWIN } \\
\text { NISBET FOREST } \\
\text { PRINCE ALBERT } \\
\text { PRINCE ALBERT } \\
\text { RAYMORE }\end{array}$} & 100 & 1 & 4 & 1 & 2 & $-5 /-10$ & $0-5$ & $10-20$ & $?$ \\
\hline & & 15 & & 3.5 & & $-11 /-5$ & $?$ & $20-25$ & cloudy, fog \\
\hline & & & & & $S$ & $-15 /-20$ & $6-8$ & $22-30$ & mostly clear \\
\hline & 50 & 25 & 1 & 6 & 1 & $-10 /-15$ & light & $5-10$ & overcast \\
\hline & 250 & 8 & 7 & 3 & 3 & $-8 /-6$ & $3-25$ & $5-15$ & overcast, fog \\
\hline \multirow{5}{*}{$\begin{array}{l}\text { REGINA } \\
\text { ROSE VALLEY } \\
\text { ST. WALBURG } \\
\text { SASKATCHEWAN FORKS } \\
\text { SASKATOON }\end{array}$} & 753 & 66.5 & 26 & 22.5 & & $-13 /-17$ & $43-17$ & $5-20$ & overcast, light snow \\
\hline & 40 & 2 & 5 & 5 & & -28 & $?$ & $?$ & mostly clear \\
\hline & 100 & 3 & 6 & 2 & & $-13 /-9$ & $40-30$ & $15-20$ & mostly cloudy, light snow \\
\hline & 46.5 & 8 & 1.5 & 3.5 & & $-10 /-11$ & $10-5$ & $10-15$ & overcast, fog \\
\hline & 547 & 117.5 & 41.5 & 52.5 & 23 & $-16 /-15$ & 24 & $8-13$ & overcast \\
\hline \multirow{5}{*}{$\begin{array}{l}\text { SCOTT } \\
\text { SEMANS } \\
\text { SKULL CREEK } \\
\text { SOMME } \\
\text { SPRING VALLEY }\end{array}$} & 224 & 10 & 7 & 4 & & -7 & $10-20$ & $10-30$ & overcast \\
\hline & 99 & 10 & 3.5 & 4 & & $-16 /-12$ & $5-2$ & $10-50$ & clear, clouding over \\
\hline & 10 & 3 & 3 & 3 & & $-5 /-1$ & $0-7$ & $8-10$ & mostly cloudy \\
\hline & 115 & 8 & 7 & $\overline{3}$ & 5 & $-5 /-8$ & $20-25$ & $25-30$ & partly cloudy \\
\hline & 25 & 16 & 1 & 3.5 & .5 & $-6 /-2$ & $8-22$ & $32-36$ & overcast, light snow \\
\hline \multirow{5}{*}{$\begin{array}{l}\text { SQUAW RAP } \\
\text { TISDALE } \\
\text { TOCO } \\
\text { WASKESIU } \\
\text { WEBB }\end{array}$} & 80 & 42 & 7 & 17 & & $-18 /-10$ & $2-20$ & $20-25$ & overcast, snow, clearing \\
\hline & 5 & & 1.5 & & 1 & $-10 /-8$ & $5-10$ & $6-7$ & mostly cloudy \\
\hline & & & & & 40 & mild & 0 & 5 & heavy hoar frost \\
\hline & 138 & 38.5 & 6 & 18 & 3 & $-12 /-9$ & $9-11$ & $15-22$ & overcast, light snow \\
\hline & 330 & 4 & 10 & 3 & & $-5 /-3$ & 0 & skiff & overcast \\
\hline & 18 & 12 & .5 & 4 & .5 & -410 & $15-0$ & $15-30$ & clear \\
\hline WHTTEBEECH & 30 & 1.5 & 1.5 & & 8 & $-12 /-14$ & $0-5$ & 25 & mostly clear \\
\hline WOLSELEY SOUTH & 100 & & 3 & & & -6 & 32 & 26 & clear \\
\hline
\end{tabular}




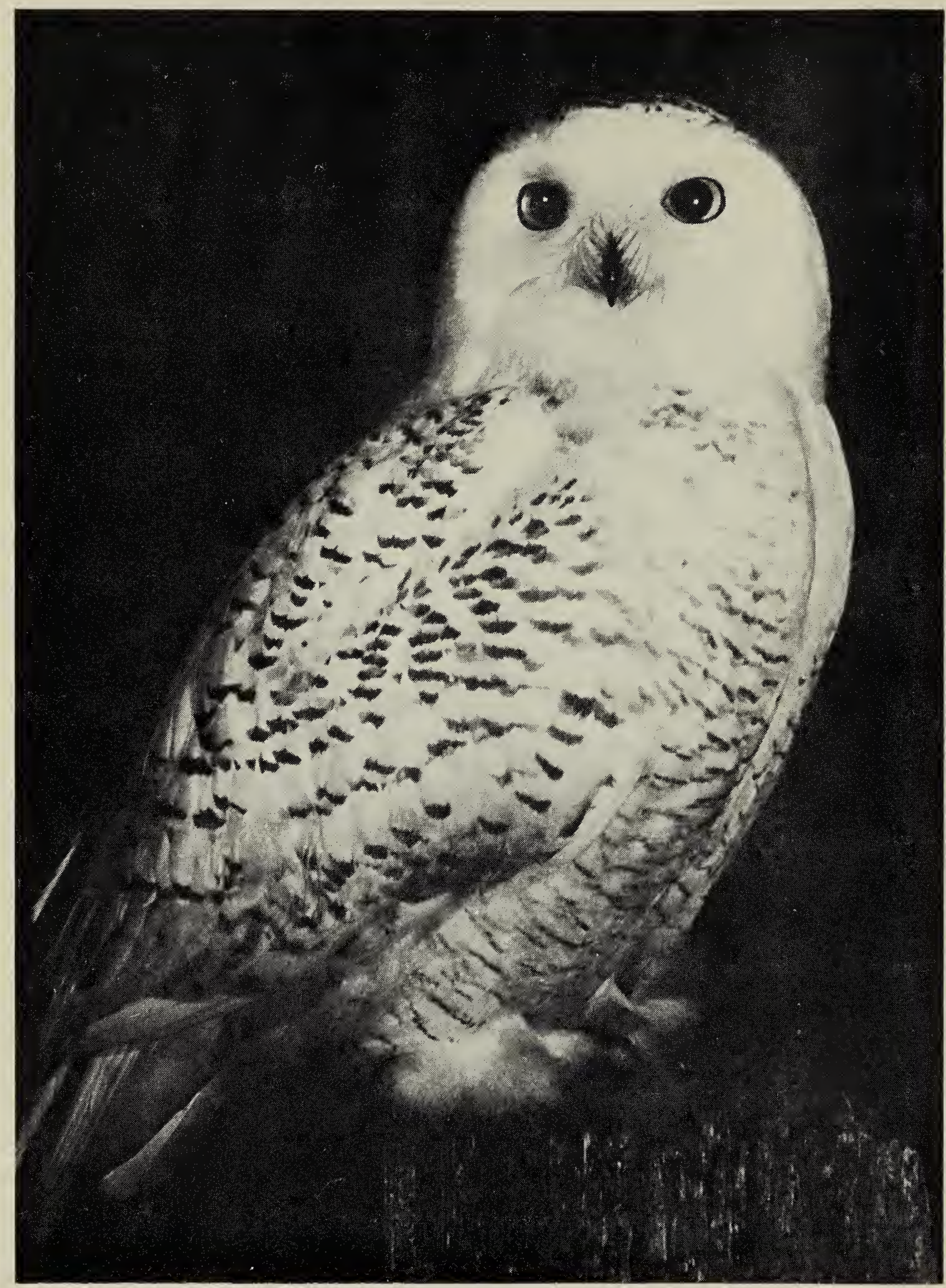




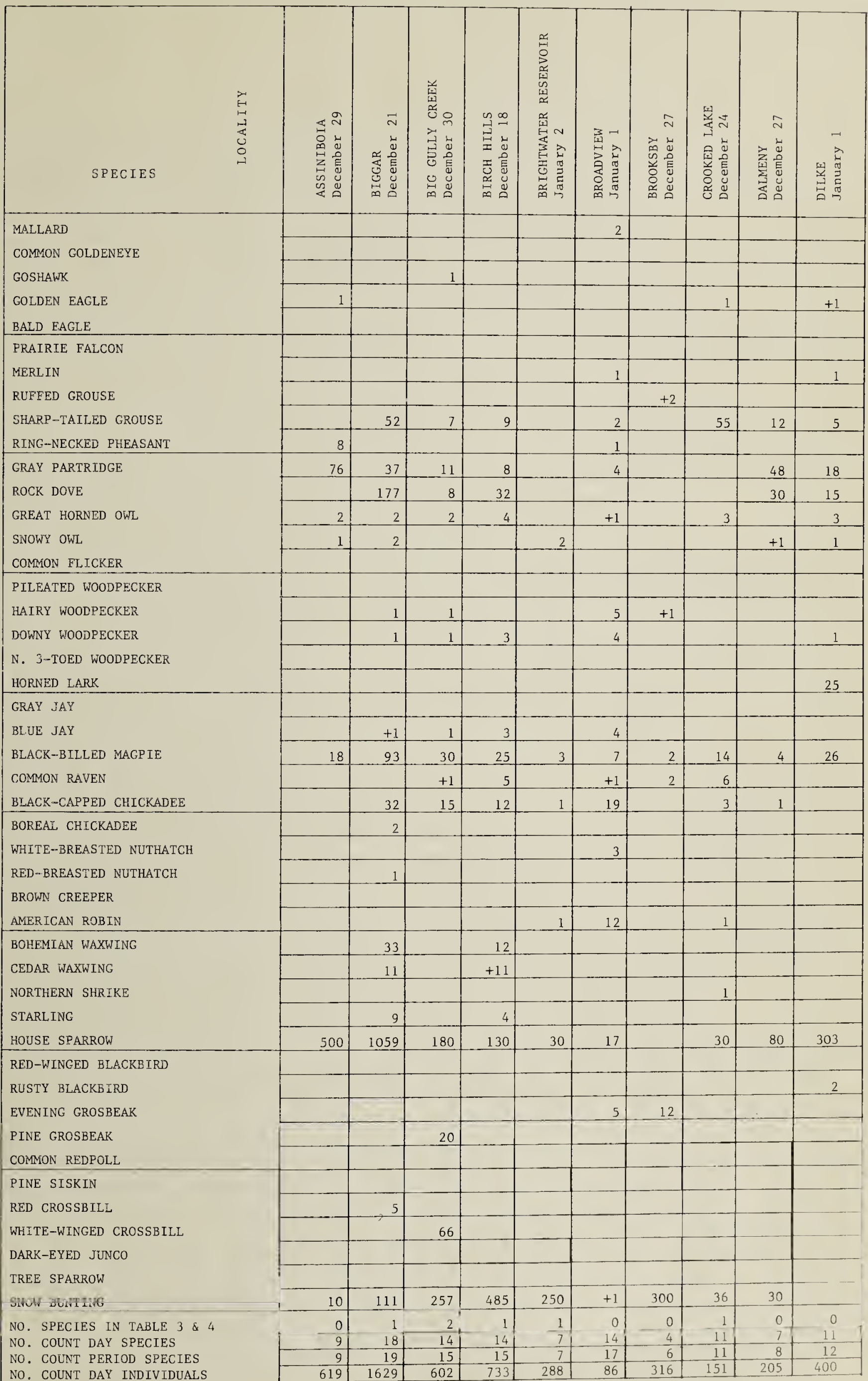




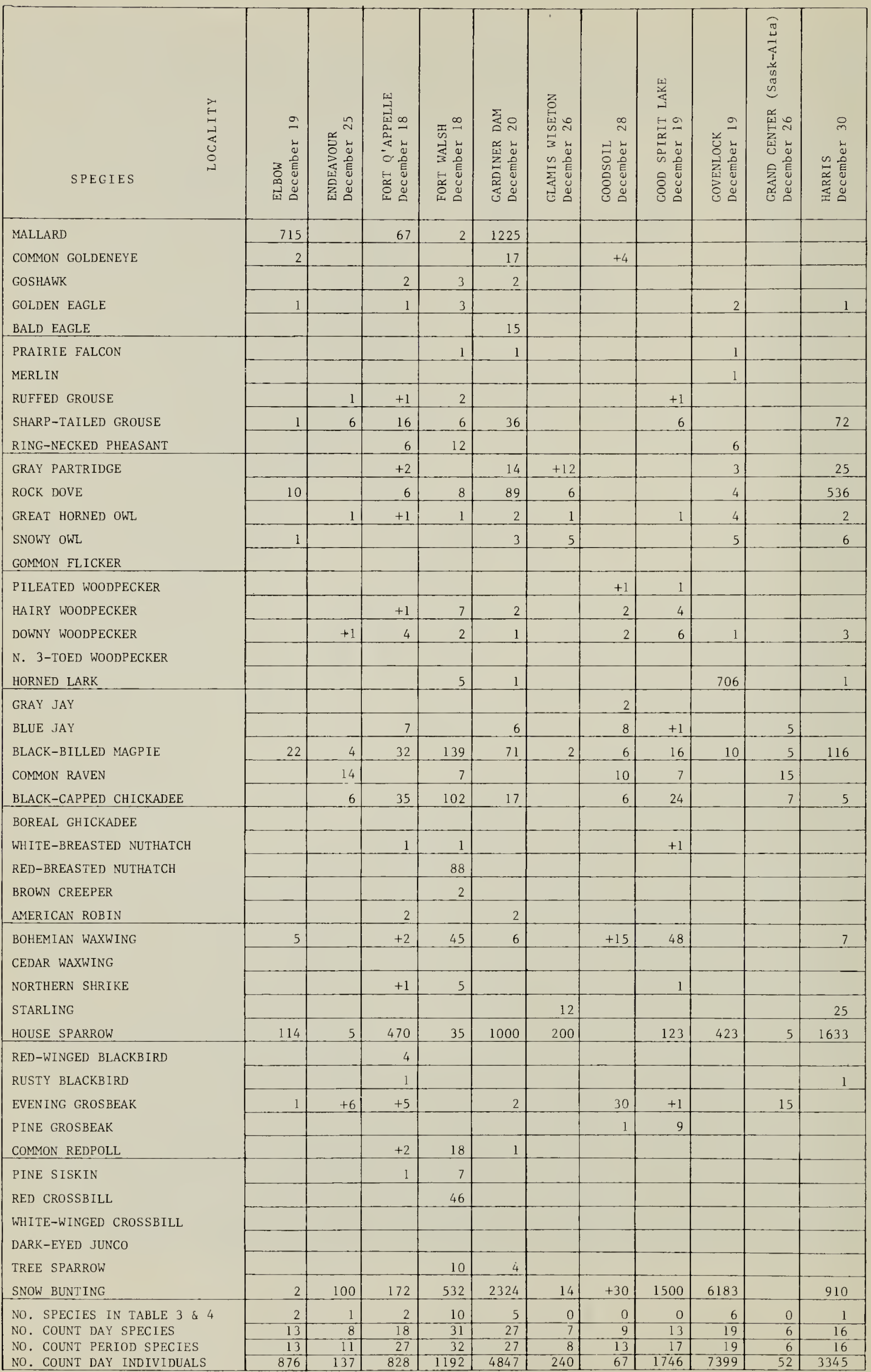


Table 2-3. SPECIES RECORDED FROM MORE THAN THREE LOCALITIES DURING COUNT PERIOD. A PLUS SIGN INDICATES A HIGH COUNT FOR A SPECIES SEEN DURING COUNT PERIOD BUT NOT ON COUNT DAY.

\begin{tabular}{|c|c|c|c|c|c|c|c|c|c|c|c|}
\hline 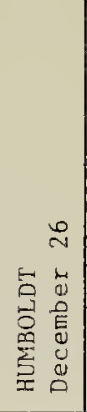 & 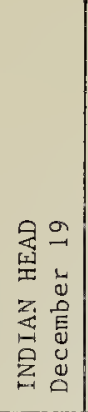 & 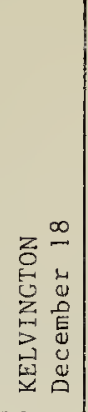 & 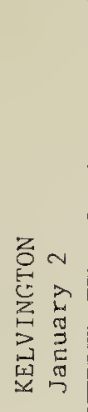 & 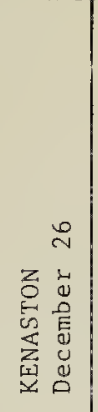 & 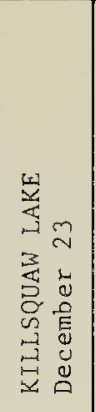 &  & 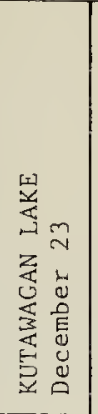 &  & 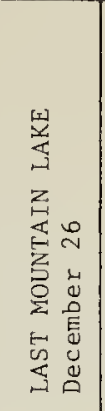 & 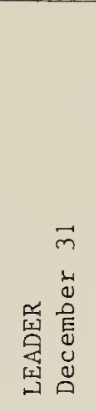 & 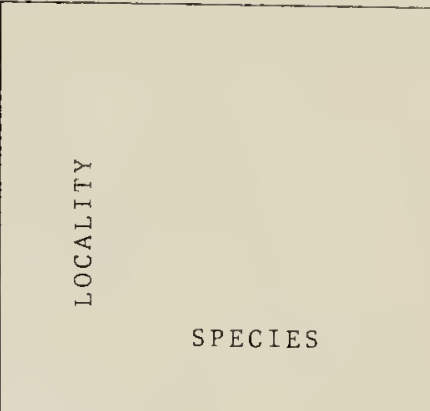 \\
\hline & 200 & & & & 22 & & & & & & \multirow{5}{*}{$\begin{array}{l}\text { MALLARD } \\
\text { COMMON GOLDENEYE } \\
\text { GOSHAWK } \\
\text { GOLDEN EAGLE } \\
\text { BALD EAGLE }\end{array}$} \\
\hline & & & & & & & & & & & \\
\hline & & & & & & & & & & & \\
\hline & 1 & & & & & & & & 1 & & \\
\hline & 1 & & & & & & & & & 1 & \\
\hline & & & & & & & & & & & \multirow{5}{*}{$\begin{array}{l}\text { PRAIRIE FALGON } \\
\text { MERLIN } \\
\text { RUFFED GROUSE } \\
\text { SHARP-TAILED GROUSE } \\
\text { RING-NECKED PHEASANT }\end{array}$} \\
\hline & & & & & & & & & & & \\
\hline & 6 & & & & & & & & & & \\
\hline+1 & 32 & 5 & +4 & 14 & & & 110 & & 137 & & \\
\hline & & & & & & & & & & & \\
\hline+8 & 17 & & +6 & 16 & & 4. & 11 & & 8 & & \multirow{5}{*}{$\begin{array}{l}\text { GRAY PARTRIDGE } \\
\text { ROCK DOVE } \\
\text { GREAT HORNED OWL } \\
\text { SNOWY OWL } \\
\text { GOMMON FLICKER }\end{array}$} \\
\hline & 11 & & 4 & 8 & 50 & 2 & 7 & & 76 & & \\
\hline+1 & 1 & & & +2 & & & 2 & & 7 & & \\
\hline & 1. & & & +2 & 1 & 1 & +1 & & 1 & & \\
\hline & & & & & & & & & & & \\
\hline & & & & & & & & & & & \multirow{4}{*}{$\begin{array}{l}\text { PILEATED WOODPEGKER } \\
\text { HAIRY WOODPECKER } \\
\text { DOWNY WOODPECKER } \\
\text { N. 3-TOED WOODPECKER } \\
\text { HORNED LARK }\end{array}$} \\
\hline+1 & 7 & 2 & 1 & \pm 1 & & & & 2 & & & \\
\hline 1 & 6 & 1 & 1 & 1 & & & 1 & & & & \\
\hline+1 & 7 & & & 4 & 6 & & 15 & & 5. & & \\
\hline & & & & & & & & 8 & & & \multirow{5}{*}{$\begin{array}{l}\text { GRAY JAY } \\
\text { BLUE JAY } \\
\text { BLACK-BILLED MAGPIE } \\
\text { GOMMON RAVEN } \\
\text { BLAGK-CAPPED GHICKADEE }\end{array}$} \\
\hline & 2 & 2 & & & & & & +3 & & & \\
\hline+10 & 35 & 4 & 9 & 15 & 6. & 1 & 27. & & 53 & 3 & \\
\hline+1 & & 1 & 8 & & & & & 46 & & & \\
\hline 5 & 38 & 3 & 7 & & 3 & & 1 & 2 & 2 & & \\
\hline & & & & & & & & 1 & & & \multirow{5}{*}{$\begin{array}{l}\text { BOREAL GHICKADEE } \\
\text { WHITE-BREASTED NUTHATGH } \\
\text { RED-BREASTED NUTHATGH } \\
\text { BROWN CREEPER } \\
\text { AMERIGAN ROBIN }\end{array}$} \\
\hline & 2 & & & & & & & & & & \\
\hline & 2 & & & & & & & & & & \\
\hline & +1 & & & & & & & & & & \\
\hline & 10 & & & & & & & & & & \\
\hline & 20 & 12 & & & 250 & & & & & 21 & \multirow{5}{*}{$\begin{array}{l}\text { BOHEMIAN WAXWING } \\
\text { CEDAR WAXWING } \\
\text { NORTHERN SHRIKE } \\
\text { STARLING } \\
\text { HOUSE SPARROW }\end{array}$} \\
\hline & & & & & & & & & & & \\
\hline & & & +1 & & & +1 & & +1 & & & \\
\hline & & & & & & & & & & & \\
\hline+100 & 495 & 4 & 200 & 150 & 65 & 50 & 267 & & 1244 & 16 & \\
\hline & & & & & & & & & & & \multirow{5}{*}{$\begin{array}{l}\text { RED-WINGED BLACKBIRD } \\
\text { RUSTY BLACKBIRD } \\
\text { EVENING GROSBEAK } \\
\text { PINE GROSBEAK } \\
\text { COMMON REDPOLL }\end{array}$} \\
\hline & & & & & & & & & & & \\
\hline & & & & & & & & 7 & & & \\
\hline+5 & & & & & & & & +3 & & & \\
\hline & & & & & & & & 7 & & & \\
\hline & & & & & & & & & & & \multirow{6}{*}{$\begin{array}{l}\text { PINE SISKIN } \\
\text { RED CROSSBILT, } \\
\text { WHITE-WINGED CROSSBILL } \\
\text { DARK-EYED JUNCO } \\
\text { TREE SPARROW } \\
\text { SNOW BUNTING }\end{array}$} \\
\hline & & & & & & & & & & & \\
\hline & & & & & & & & & & & \\
\hline & & & 1 & & & & & & & & \\
\hline & & & & & & & & & & & \\
\hline+50 & 270 & 50 & 300 & 18 & 5 & 100 & 9483 & & 12944 & 3 & \\
\hline 0 & 0 & 0 & 0 & 0 & 0 & 0 & 0 & 0 & 1 & 0 & \multirow{4}{*}{$\begin{array}{l}\text { NO. SPECIES IN TABLE } 3 \& 4 \\
\text { NO. GOUNT DAY SPEGIES } \\
\text { NO. GOUNT PERIOD SPECIES } \\
\text { NO. COUNT DAY INDIVIDUALS }\end{array}$} \\
\hline 2 & 21 & 10 & 9 & 8 & 9 & 6 & 10 & 7 & 11 & 5 & \\
\hline 12 & 22 & 10 & 12 & 11 & 9 & 7 & 11 & 10 & 11 & 5 & \\
\hline & & & & & 408 & 158 & 9924 & & 14479 & 44 & \\
\hline
\end{tabular}


Table 2-4.

SPECIES RECORDED FROM MORE THAN THREE LOCALITIES DURINC COUNT PERIOD. A PLUS SIGN INDICATES A HICH COUNT FOR A SPECIES SEEN DURINC COUNT PERIOD BUT NOT ON COUNT DAY.

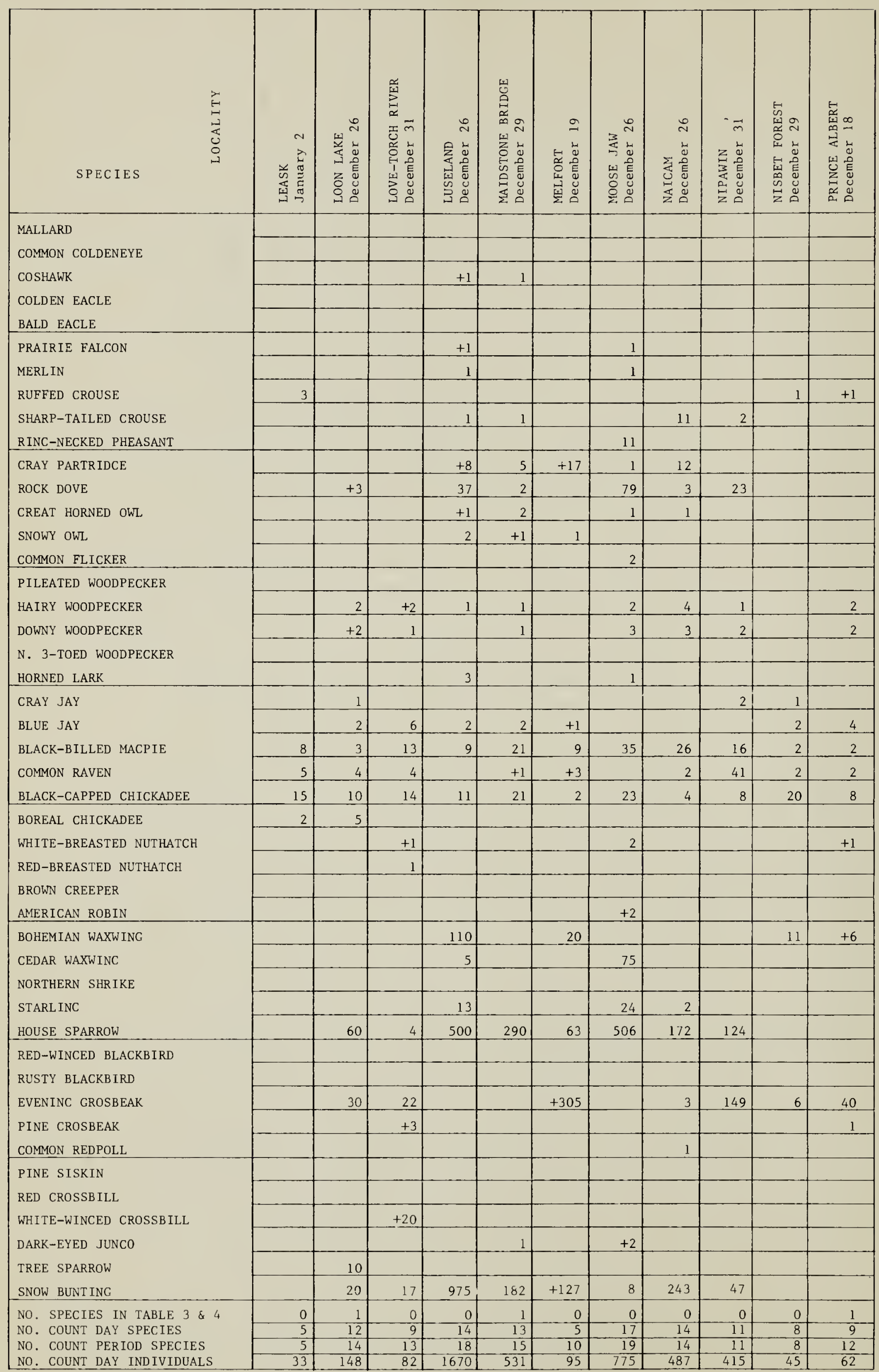




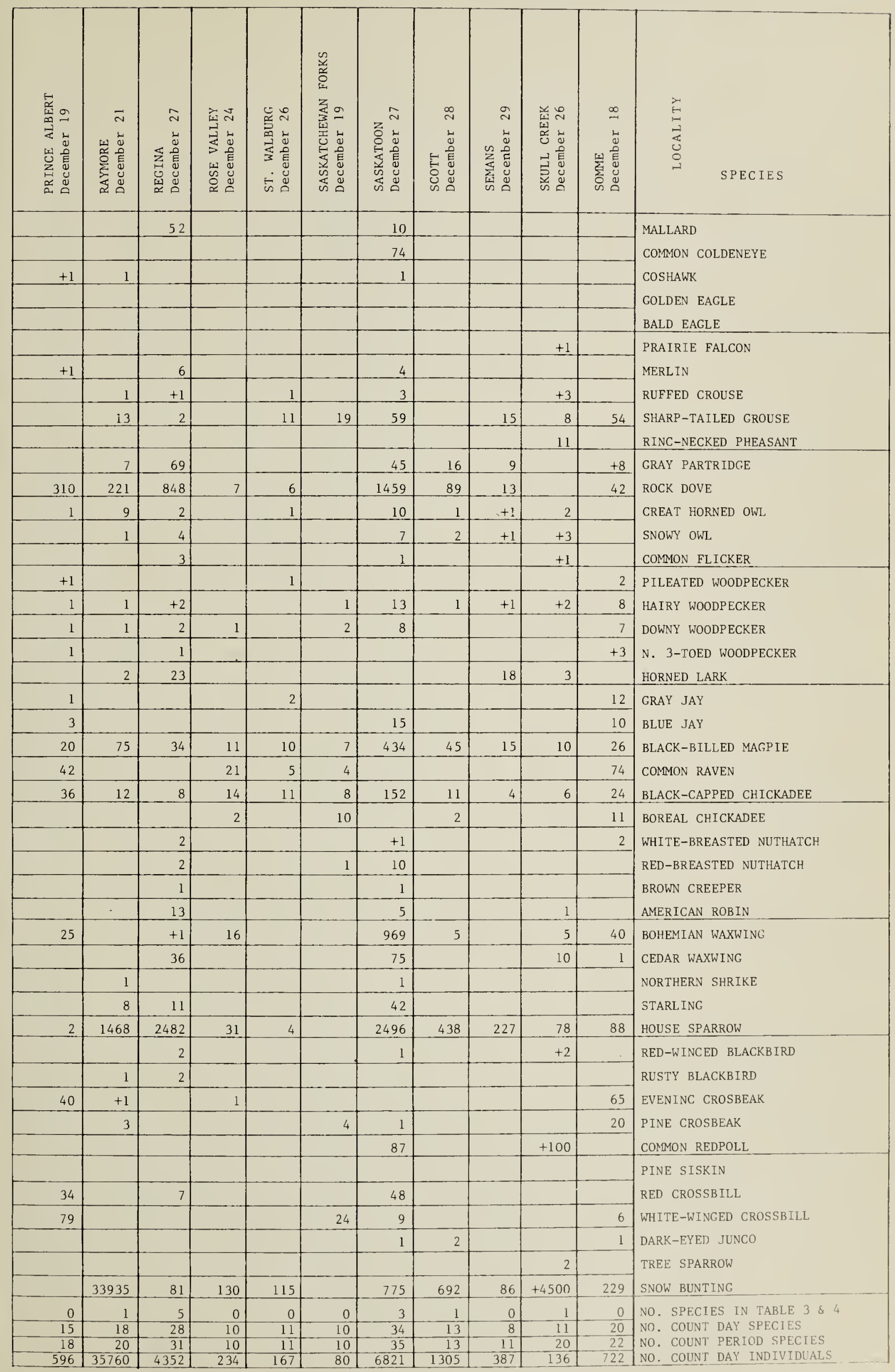


Table 2-6.

SPECIES RECORDED FROM MORE THAN THREE LOCALITIES DURINC COUNT PERIOD. A PLUS SICN INDICATES A HIGH COUNT FOR A SPECIES SEEN DURING COUNT PERIOD BUT NOT ON COUNT DAY.

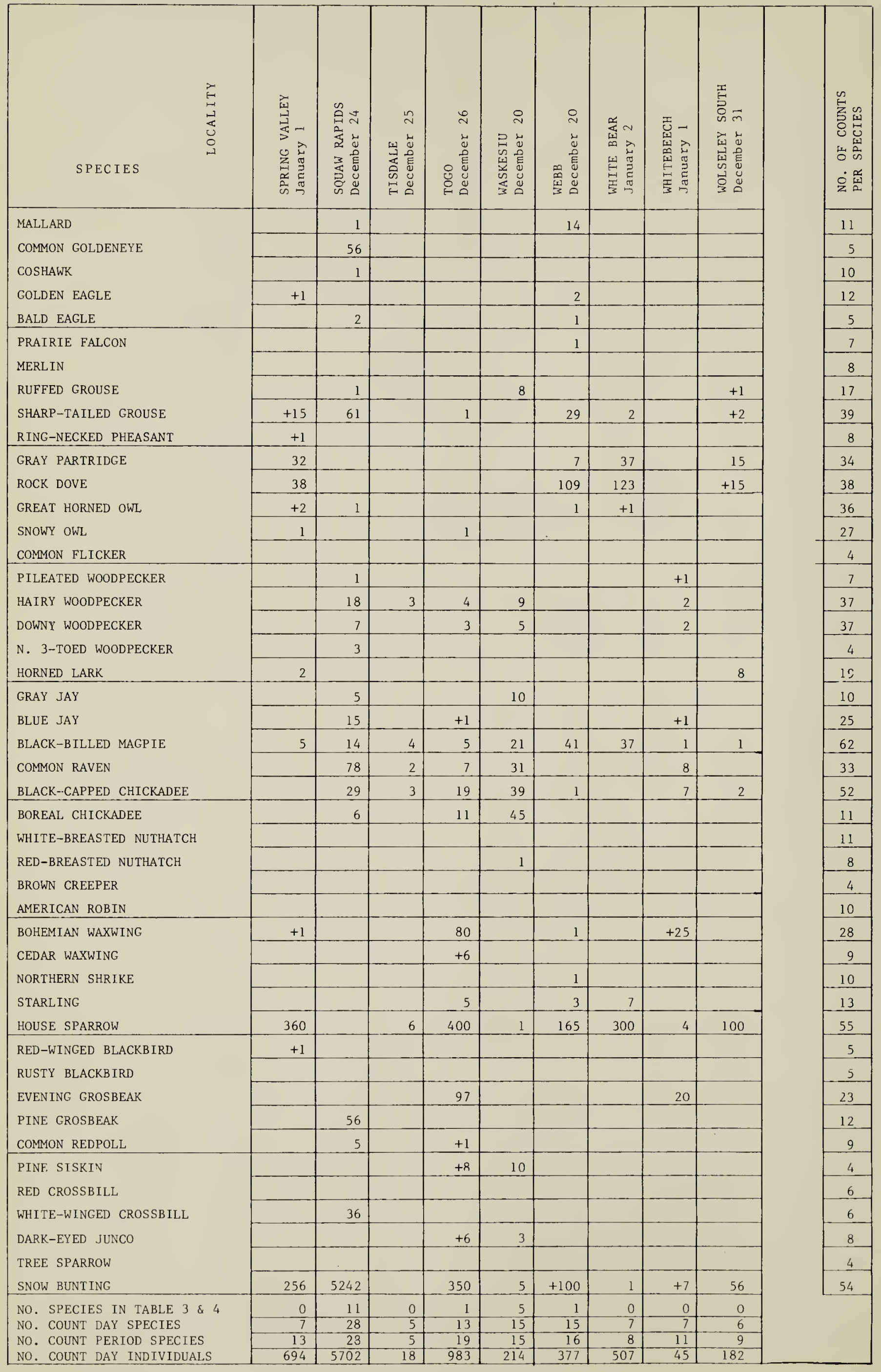




\begin{tabular}{|c|c|}
\hline SPEGIES & LOGALITIES AND NUMBERS SEEN \\
\hline \multirow{5}{*}{$\begin{array}{l}\text { GOMMON LOON } \\
\text { GANADA GOOSE } \\
\text { GADWALI } \\
\text { COMMON MERGANSER } \\
\text { RED-BREASTED MERGANSER }\end{array}$} & GARDINER DAM, 1 . \\
\hline & REGINA, 623 . \\
\hline & GARDINER DAM, 1 \\
\hline & GARDINER DAM， 2; SASKATOON，1; SQUAW RAPIDS, 5. \\
\hline & GARDINER DAM, 1. \\
\hline \multirow{5}{*}{$\begin{array}{l}\text { SHARP-SHINNED HAWK } \\
\text { COOPER'S HAWK } \\
\text { RED-TAILED HAWK } \\
\text { ROUGH-LEGGED HAWK } \\
\text { GYRFALCON }\end{array}$} & GROOKED LAKE, 1. \\
\hline & SKULL GREEK, + 1 \\
\hline & SQUAW RAPIDS, 1. \\
\hline & FORT WALSH, +1 ; GOVENLOCK, 2 ; RAYMORE, +1. \\
\hline & ELBOW, 1 ; SQUAW RAPIDS, 3 ; TOGO, +1. \\
\hline \multirow{5}{*}{$\begin{array}{l}\text { PEREGRINE FALCON } \\
\text { SPRUGE GROUSE } \\
\text { SAGE GROUSE } \\
\text { CHUKAR } \\
\text { TURKEY }\end{array}$} & WEBB, 1. \\
\hline & WASKESIU, 1. \\
\hline & GOVENLOCK, 7. \\
\hline & GOVENLOCK, 1 . \\
\hline & FORT WALSH, 5. \\
\hline \multirow{5}{*}{$\begin{array}{l}\text { KILLDEER } \\
\text { COMMON SNIPE } \\
\text { HAWK OWL } \\
\text { GREAT GRAY OWL } \\
\text { BOREAL OWL }\end{array}$} & GOVENLOGK, 1 . \\
\hline & BRIGHTWATER RESEVOIR, 1 ; FORT WALSH, 1 . \\
\hline & BIRGH_HILLS, 1 ; LOON LAKE, I; SQUAW RAPIDS, 1. \\
\hline & SQUAW RAPIDS, 2. \\
\hline & SQUAW RAPIDS, 1. \\
\hline \multirow{5}{*}{$\begin{array}{l}\text { BLACK-BAGKED THREE TOED WOODPECKER } \\
\text { COMMON CROW } \\
\text { GOLDEN-GROWNED KINGLET } \\
\text { LOGGERHEAD SHRIKE } \\
\text { BREWER'S BLACKBIRD }\end{array}$} & SCOTT, 1 ; SQUAW RAPIDS, 2. \\
\hline & REGINA, 1 . \\
\hline & BIGGAR, 1; FORT WALSH, 15; WASKESIU, 2. \\
\hline & REGINA, 1 . \\
\hline & FORT QU'APPELLE, 1 ; SASKATOON, 3. \\
\hline \multirow{7}{*}{$\begin{array}{l}\text { PURPLE FINCH } \\
\text { HOARY REDPOLL } \\
\text { HARRIS' SPARROW } \\
\text { WHITE--THROATED SPARROW } \\
\text { SONG SPARROW } \\
\text { MCGOWN'S LONGSPUR } \\
\text { LAAPLAND LONGSPUR }\end{array}$} & SASKATOON, 1. \\
\hline & FORT WALSH, 15. \\
\hline & FORT WALSH, 1. \\
\hline & HARRIS, 2 ; PRINCE ALBERT, 1; REGINA, 1. \\
\hline & FORT QU'APPELLE, +1 \\
\hline & GOVENLOCK, 1 . \\
\hline & GOVENLOGK, 38. \\
\hline
\end{tabular}

Table 4. BIRDS NOT IDENTIFIED TO SPECIES.

\begin{tabular}{|c|c|}
\hline & LOGALITIES AND NUMBERS SEEN \\
\hline \multirow{6}{*}{$\begin{array}{l}\text { SWAN species } \\
\text { BUTEO species } \\
\text { EAGLE species } \\
\text { FALGON species } \\
\text { GROUSE species } \\
\text { WOODPECKER species }\end{array}$} & ELBOW, 1 \\
\hline & FORT WALSH, 1. \\
\hline & LAST MOUNTAIN LAKE, 1. \\
\hline & GARDINER DAM, 1. \\
\hline & BIG GULLY GREEK, 1; SQUAW RAPIDS, 1 . \\
\hline & FORT WALSH, 2; MAIDSTONE BRIDGE, 1 ; SQUAW RAPIDS, 5; WASKESIU, 1. \\
\hline \multirow{6}{*}{$\begin{array}{l}\text { THREE-TOED WOODPEGKER species } \\
\text { GHICKADEE species } \\
\text { NUTHATGH species } \\
\text { WAXWING species } \\
\text { REDPOLL species } \\
\text { CROSSBILL species }\end{array}$} & BIG GULLY GREEK, 1 . \\
\hline & SQUAW RAPIDS, 1 ; WASKESIU, 21. \\
\hline & ENDEAVOUR, +1 ; WASKESIU, 1 . \\
\hline & REGINA, 33. \\
\hline & FORT WALSH, 52; SQUAW RAPIDS, 46. \\
\hline & FORT WALSH, 11. \\
\hline
\end{tabular}

